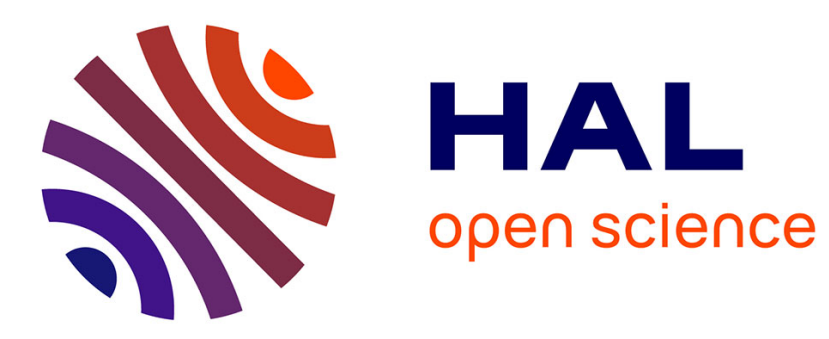

\title{
Spectralités et ancêtres : traces de l'inquiétante étrangeté dans les représentations de la guerre de libération nationale au Zimbabwe
}

Elara Bertho

\section{- To cite this version:}

Elara Bertho. Spectralités et ancêtres : traces de l'inquiétante étrangeté dans les représentations de la guerre de libération nationale au Zimbabwe. Trans: Revue de Littérature Générale et Comparée, 2017, 21, 10.4000/trans.1565 . hal-01793429

\section{HAL Id: hal-01793429 \\ https://hal.science/hal-01793429}

Submitted on 16 May 2018

HAL is a multi-disciplinary open access archive for the deposit and dissemination of scientific research documents, whether they are published or not. The documents may come from teaching and research institutions in France or abroad, or from public or private research centers.
L'archive ouverte pluridisciplinaire HAL, est destinée au dépôt et à la diffusion de documents scientifiques de niveau recherche, publiés ou non, émanant des établissements d'enseignement et de recherche français ou étrangers, des laboratoires publics ou privés. 


\section{TRANS-}

Revue de littérature générale et comparée

\section{Spectralités et ancêtres : traces de l'inquiétante étrangeté dans les représentations de la guerre de libération nationale au Zimbabwe}

Ghostly figures and ancestors: traces of the unheimlich in representations of the Zimbabwe War of Liberation

\section{Elara Bertho}

\section{revues.org}

\section{Éditeur}

Presses Sorbonne Nouvelle

Édition électronique

URL : http://trans.revues.org/1565

DOI : $10.4000 /$ trans. 1565

ISSN : 1778-3887

\section{Référence électronique}

Elara Bertho, «Spectralités et ancêtres : traces de l'inquiétante étrangeté dans les représentations de la guerre de libération nationale au Zimbabwe », TRANS- [En ligne], | 2017, mis en ligne le 17 mars 2017, consulté le 06 avril 2017. URL : http://trans.revues.org/1565 ; DOI : 10.4000/trans.1565

Ce document a été généré automatiquement le 6 avril 2017.

Tous droits réservés 


\title{
Spectralités et ancêtres : traces de l'inquiétante étrangeté dans les représentations de la guerre de libération nationale au Zimbabwe
}

\author{
Ghostly figures and ancestors: traces of the unheimlich in representations of \\ the Zimbabwe War of Liberation
}

Elara Bertho

1 Les personnages de l'ancêtre revenant conseiller les vivants, des mudzimu en shona (ancêtres de la lignée), croisent le motif du double - celui qui est de la même famille et qui pourtant n'est pas moi - fonctionnant sur le modèle du miroir, et le motif du mort-vivant - celui dont la mort ne passe pas. Fantasme infantile et fantasme de mort, les deux sont unheimlich ${ }^{1}$ d'après Freud dans son essai sur Hoffman. Chez les romanciers zimbabwéens, l'ensemble des intrigues racontant la guerre de libération nationale se situent toutefois dans des univers parfaitement réalistes, et ne sont donc pas des « contes de fées » au sens où l'a pris Freud pour L'homme au sable.

2 Quelques éléments de contexte s'imposent sur la guerre de Libération nationale au Zimbabwe, que Chenjerai Hove décrit dans ses ouvrages. En 1965, la Rhodésie déclare son indépendance à l'égard de la Grande Bretagne, de manière unilatérale. Un gouvernement d'apartheid, extrêmement dur, est mis en place, instituant de manière violente des migrations de populations, et des encadrements dans des réserves, aux sols très peu fertiles ${ }^{2}$.

3 Deux fronts politiques et combattants émergent : le ZANU, à majorité shona, et le ZAPU à majorité ndebele, les deux ayant par ailleurs des bras armés qui ont mené une guérilla pendant quinze ans. En 1980, porté par le ZANU, Robert Mugabe arrive au pouvoir, plébiscité par le premier scrutin non racial de l'histoire du Zimbabwe. Il y reste au pouvoir, jusqu'à aujourd'hui, après avoir mené des réformes agraires visant à répartir les 
richesses et les grandes propriétés terriennes. Une dévaluation a entraîné la faillite de la monnaie, qui n'a plus cours désormais. Le gouvernement rhodésien, mené par Ian Smith, a animé une intense propagande, accusant les combattants d'être des terroristes. Ceux-ci ont mené une contre-propagande, en militant pour les droits civiques, et en dénonçant le système de ségrégation raciale de l'apartheid, les inégales répartitions de richesses, et les exploitations agricoles et minières.

4 L'écriture de la violence extrême de la guerre de maquis est un thème majeur de la littérature zimbabwéenne. Yvonne Vera, dans Nehanda, The Stone Virgins ${ }^{3}$, Stanlake Samkange avec On Trial for my Country ${ }^{4}$, Year of the Uprising ${ }^{5}$, en font un cadre de leurs récits, en le reliant à l'histoire de la colonisation : la Chimurenga ("révolte»), est suivie d'une Seconde Chimurenga, qui amènera l'indépendance. Ce saut chronologique, entre l'histoire des résistants à la colonisation de la fin du XIX ${ }^{\mathrm{e}}$ siècle et la guerre de libération des années 1970, est établi dans les textes, en s'appuyant sur une rhétorique de propagande diffusée par les deux partis de l'opposition. Nous proposons une lecture des ouvrages de Chenjerai Hove, qui présentent l'originalité de jouer avec les points de vue l'étrangeté de la guerre. Chenjerai $\mathrm{Hove}^{6}$ est poète, romancier, essayiste et journaliste. Ayant étudié à l'Université d'Afrique du Sud, puis du Zimbabwe, il est également professeur. Il écrit en anglais et en shona, principalement autour de la guerre de libération nationale de la Rhodésie, de 1965 à 1980, date à laquelle elle devient Zimbabwe. Très critique à l'égard de la politique de Robert Mugabe, il termine sa vie en exil, reçu à Brown University, dans le Rhode Island. Chez Hove, les guerres de maquis sont vues à travers les perceptions villageoises, d'intrusions de la bataille par irruptions ponctuelles, irrégulières, qui trouent le texte, sur le fond oppressant de l'exploitation agricole par les Blancs.

Publié à Harare en 1988, Ossuaire (Bones) ${ }^{7}$ présente un cas d'écriture de la guerre de libération particulièrement intéressant. Marita pleure le départ de son unique fils au combat, enrôlé de gré ou de force. Son mari Marume lui reproche son départ et maudit leur union. Marita se réfugie auprès de Janifa, la fiancée de son fils, et lui annonce qu'elle partira bientôt en ville à la recherche de son fils. Sur la plantation, Manyepo fait régner un climat de terreur et menace de représailles ceux dont les enfants ont déserté les champs pour la guerre. Chisaga, le cuisinier, jouit d'un statut de privilège dans ce système d'exploitation. Marita est battue parce que son fils a disparu. Elle part quelques temps plus tard, et meurt sans avoir retrouvé trace de son fils. Janifa, violée par Chisaga, avec la complicité de sa propre mère, devient progressivement folle, et est enfermée dans un asile, où son fiancé la retrouve, sans que l'on sache si elle guérira ou non. Le titre fait allusion à un fait historico-légendaire bien connu au Zimbabwe: une femme médium, prêtresse, nommée Nehanda, aurait mené une partie des révoltes contre les colons en 1896. Au moment d'être pendue en 1897, elle aurait prophétisé son retour et annoncé la chute des Blancs en s'écriant « My bones shall rise again $»^{8}$. Les combattants de la seconde Chimurenga se sont reconnus être les «os » de Nehanda, renaissant de la terre, pour venir venger leurs ancêtres, en chassant les colons de leurs territoires. Bones fait ainsi référence à la fois aux charniers des victimes de la guerre de libération nationale, aux ossements de Nehanda, mais aussi au symbole d'espoir des combattants qui se sont battus pour une dignité civique et politique. Il y a d'emblée un double horizon de la spectralité dans le texte: d'une part, l'horizon personnage-fantôme (celui des personnages qui se vident de leur substance par la douleur, l'horreur, l'épuisement), d'autre part, l'horizon de l'ancêtre (celui d'une ombre qui parle et qui fait lever les os des disparus). 
6 Nous proposons de dégager plusieurs niveaux de lecture de l'écriture de la violence coloniale, à travers la notion d'inquiétante étrangeté :

La fascination et la terreur dominent le récit : une lecture symbolique de l'histoire et de la violence constitue le premier niveau de lecture.

Un second niveau, que Freud nomme le « retour du refoulé » : ce qui doit être caché qui remonte (les seins, le cadavre, la maison des fous). Le corps est caché et montré à la fois (la scène du viol et de la folie de Janifa devenue impudique à la fin, tout comme la femme qui cherche le corps de Marita et qui menace de montrer ses parties génitales : ces femmes sont dans un au-delà des normes de la pudeur).

Enfin, narrativement, c'est le texte même qui est bouleversé par l'insertion de la voix des ancêtres : Hove fait advenir une polyphonie narrative, qui fait intervenir également la voix des ancêtres, dans deux chapitres intitulés précisément « Les ancêtres parlent ». Il y a là une rupture de continuité narrative réaliste, et des imbrications de voix multiples qui ont des effets d'interprétations intéressants.

7 Nous verrons dans cet article, à partir de plusieurs études de textes, comment se manifeste l'inquiétante étrangeté dans la narration de la guerre de libération. À travers le roman polyphonique, par certains moments choral, Hove décrit d'abord les rumeurs liées à la figure des terroristes - du nom donné aux révolutionnaires par le gouvernement. Ces figures de l'étrange - qui tuent et épouvantent la population - sont rejetées dans le domaine de l'automate, de l'inhumain, du hors-la-loi, de l'horreur, tout en étant toujours assimilées au foyer, à l'intime, ce qui les rend d'autant plus dangereux : ce sont des jeunes hommes d'ici, des "fils de la terre ", qui ont "mal tourné », et la rumeur se nourrit de cette étrange proximité avec l'intime. Progressivement, par couches successives de récits, de contes, d'anecdotes, l'inquiétant se décentre, et ne porte non plus sur les terroristes, mais sur la situation d'exploitation coloniale dans son ensemble, ce qui amène le lecteur à changer de regard sur ces mêmes terroristes. C'est ce mouvement de balancier de l'étrange et du unheimlich opéré par la narration que nous voudrions explorer ici.

\section{La guerre civile, le terrorisme : I'horreur dans la maison}

8 La guerre civile, le maquis, le terrorisme : l'imaginaire en jeu est celui d'une guerre où tout est possible, où l'horreur peut s'abattre à n'importe quel moment dans la maison, dans le heimlich, dans le lieu très sûr du foyer, de l'âtre. Par définition, c'est une guerre où il n'y a pas de champ de bataille, puisqu'il peut être, en puissance, n'importe où, et qu'il n'épargne pas le corps domestique du foyer. Les victimes «civiles » sont le cœur du conflit par excellence, et les cibles privilégiées, par un retournement cynique de l'objet des massacres.

9 Mais plus inquiétant certainement, ce qui est particulièrement frappant dans le terrorisme, est que l'agresseur provient lui-même de ce foyer qu'il attaque : il fait corps et appartient au heimlich que pourtant il s'attache à massacrer.

Julia Kristeva, dans Étrangers à nous-mêmes 9 , souligne à quel point heimlich et unheimlich sont liés, et comment l'étrange gît toujours-déjà, tapis, caché, recroquevillé, au cœur du familier. C'est le propre du terrorisme que de frapper à partir de l'intime: ce sont les jeunes des cités françaises et belges qui attaquent aujourd'hui les capitales européennes.

11 L'ouverture du roman met en scène une femme, Marita, demandant à Janifa de lui relire la lettre d'amour que son fils lui avait adressé avant de partir s'engager dans la guérilla. 
La terreur est mise en scène à partir d'une circulation de voix étrangères, de récits, de mythes et de légendes qui déshumanisent la figure de l'horreur, du combattant, en soulignant les meurtres et les exactions - qui ont été réelles, comme en témoignent les romans d'Yvonne Vera, par exemple les viols dans Without A Name ${ }^{10}$. S'élabore dans toute la première partie du texte une "poétique de la rumeur ", pour reprendre l'expression de Xavier Garnier ${ }^{11}$, où ces enfants partis dans les maquis sont transformés en monstres, hors sol.

Oui, j'ai la lettre, même si je sais que je ne l'épouserai jamais. Comment épouseraisje un terroriste? Ne raconte-t-on pas qu'un terroriste dévore les gens tout cru? $\mathrm{Ne}$ dit-on pas qu'un terroriste prend la femme d'autrui, et couche avec sous les yeux du mari, avant de demander aux parents de lui faire cuire leurs enfants? Je ne peux pas épouser un terroriste, un assassin qui tue sa propre mère.

- Mais c'est mon fils, dit-elle en pleurant doucement. Je ne lui ai pas appris à manger les autres. Ni à coucher avec toutes les femmes croisées sur le chemin du puits. (p. 16)

Passant de bouche en bouche, les récits entourant les terroristes, ces disparus, enflent, gonflent, et rejoignent les contes et légendes de la tradition orale, selon des procédés de prolifération de «rumeurs urbaines» qu'a étudiées Julien Bonhomme ${ }^{12}$ : des acteurs centralisent et diffusent des récits, avec des réseaux de canalisation et d'endiguement, semblables à ceux des épidémies ${ }^{13}$ (« ne dit-on pas ... », « on disait que ... »).

Or dans cet extrait, ce qui nous semble intéressant, c'est comment, d'emblée, Marita lutte pour réintégrer son fils dans le cercle de l'humain (selon une logique de glissement avec la réplique de Janifa : « tue sa propre mère »/ « mon fils »).

N'as-tu pas entendu ce qu'on raconte de ce que peuvent faire les combattants? Je ne crois pas qu'on dise tout le temps la vérité. Il faudrait le voir soi-même de ses yeux pour le croire parfois. Ventre maternel en porte de toute nature : voleurs ou prêtres. Mais je n'ai pas appris à mon garçon à mal agir, parce que le faire, c'est s'humilier devant tout le village. Alors comment a-t-il pu me quitter et se mettre à voler et à commettre toutes sortes de noirceurs que la bouche a honte de nommer? C'est pour cela aussi que je veux le retrouver. J'ai envie de lui demander si c'est vrai ce qu'on rapporte. Ça pourrait être vrai comme dans les histoires que nous racontaient nos grands-parents... les histoires d'ogres aux dents longues qui se repaissent du sang des braves jusqu'à ce qu'il n'y en ai plus un seul en vie. [...] C'est peut-être de la folie, pourtant ça s'est produit. Mais que des enfants mangent leurs pères, c'est une question qui requiert un puissant guérisseur. [...] (p. 74)

14 Ce mélange de familier et d'horreur est souligné par le proverbe sur le ventre maternel, pouvant enfanter à la fois le bon et le mauvais. La question de la rumeur et du conte ressurgit toujours pour caractériser les combattants.

Tant pis si on te traitait de Mère des Terroristes! Tu te contentais de sourire, comme heureuse d'avoir engendré des enfants qui faisaient griller d'autres enfants pour les manger. Des enfants dont on disait qu'ils étaient si cruels qu'ils pouvaient jeter un homme dans de grandes flammes où il se consumait; et eux dansaient autour du feu comme les monstres dont tu m'as parlé un jour dans l'histoire des filles qui, sans le savoir, poursuivaient des hommes-lions [man-lion: mhondoro, en shona]. Mais Marita, as-tu retrouvé ton fils là-bas? (p. 37)

Dans cet autre passage, entre un mari et une femme (Marume et Marita), la femme se bat contre son mari pour conserver une humanité aux terroristes, en les ramenant à «la terre ", comme protection maternelle universelle :

Les enfants sont venus et ils ont été tués.

- Quels enfants? Es-tu folle?

- Les enfants. Ils ont été tués. Mais on n'a pas dit ce qu'ils voulaient. Ils ont été tués. 
- La folie te ronge la pensée. Des voleurs, des gangsters, des bandits armés, oui, comment peux-tu les appeler « les enfants »? Les enfants de qui, on peut savoir?

- Les enfants de la terre.

- C'est l'esprit Zapu qui te possède ! L'esprit du mal ! (p. 90)

Le ZAPU, du nom du parti politique de la rébellion, est transformé en injure par le mari, de toute évidence hostile à la guerre de libération nationale.

La douleur de la perte de l'enfant, la honte et la culpabilité qui l'accompagnent, creusent le personnage de la mère jusqu'à en faire un personnage vidé par ce clivement, rendu fantômatique, spectral, dénué d'intériorité propre. Ainsi interroge Janifa :

«Marita, votre cœur est empli de votre fils. N'y a-t-il rien d'autre en votre cœur que votre fils? lui dis-je.

- De quoi d'autre veux-tu que je l'emplisse? Que puis-je y mettre d'autre?»

Je bredouille :

«Je ne sais pas. Mais vous êtes forte, très forte.

- Si je l'étais, j'emplirais mon cœur d'autre chose. »

Elle grimace avant que son visage devienne à mes yeux une ombre [shadow]. Elle semble parler avec la terre, avec les grains de sable derrière cette termitière. (p. 26-27)

Comme le souligne Xavier Garnier à propos de la notion de figure ${ }^{14}$, les personnages romanesque décrits dans les situations impériales se creusent par l'intérieur, se vident de leur substance pour devenir des êtres inquiétants par leur absence de consistance, mais qui interrogent davantage le lecteur. Ces êtres fantômes vivent avec leurs propres fantômes, au sens où Derrida, dans Spectres de Marx ${ }^{15}$, appelle à " apprendre à vivre avec " les disparus, dans leur «compagnonnage». Marita refuse de "vivre avec»: elle est creusée de l'intérieur par le manque de son fils, elle se dissout jusqu'à devenir, vivante, un ancêtre (shadow). Chenjerai Hove, par ailleurs, a rédigé un ouvrage du même titre ${ }^{16}$, qui réfère aux ancêtres. Pour Janifa, cette présence spectrale de Marita se dissolvant de douleur devient peu à peu une figure tutélaire à laquelle elle s'adresse, comme à un ancêtre. Elle continuera d'ailleurs son dialogue avec elle, même après sa mort.

\section{Le seuil de l'étrange : retournement de l'objet de l'inquiétude}

19 Cette dissolution est peu à peu jointe à celle du travail dans les plantations, qui engage une désindividuation progressive :

À la fin de sa journée de travail, Marita ressemble à un fantôme [ghost] dans les champs. Manyepo appelle cela travailler dur. Mais quand je songe que Marita ne reçoit à manger qu'un bol de haricots parce qu'elle n'a pas d'enfants, à l'intérieur ça me fait mal. Ce qui se passe à l'intérieur, il est difficile de le comprendre tout le temps. (p. 74-6)

Marita a longtemps eu du mal à procréer, et son unique enfant est parti à la guerre. Pour cette faute, elle est traitée comme une femme sans enfant par le propriétaire de la plantation, qui la rémunère comme si elle n'avait jamais eu d'enfant. Cette blessure, et ce déni de ses droits, est douloureusement vécu par Janifa, dont la plainte se révèle sans écho, dans le village où elles vivent.

La déshumanisation par le travail dans les champs engage bientôt, par touches successives, l'ensemble des travailleurs. Ainsi Marita raconte-t-elle à Janifa le quotidien de son frère : 
Il faut voir les coupeurs de canne se lever tôt le matin, quand rien ne bouge encore, et les voir rentrer l'après-midi : ils ressemblent à des arbres calcinés qui auraient des jambes et des yeux. Tu sais qu'on brûle la canne avant de la couper, pour effrayer les gros serpents, à ce qu'on dit. Mais du coup, le corps des coupeurs en devient noir comme charbon de bois. Quand tu vois sur le fils de ta mère tout ce noir de fumée, tu pleures. Oui, aussitôt tu pleures. Les larmes viennent toutes seules et ton cœur saigne. (p. 114) cuisine pour le Blanc, donc qui ne travaille pas dans les champs. Il pourrait l'empoisonner tous les jours, et pourtant il ne le fait pas. Il est son homme de confiance, il le voit à chaque repas, il recueille ses confidences, il le porte dans son lit lorsqu'il s'enivre. Cette figure est dupliquée, par des contes que transmet Marita à Janifa, à l'échelle du pays cette fois :

Derrière ces montagnes, disait Marita, la terre est devenue rouge et les gens ont commencé à voir du sang partout. Les arbres eux-mêmes avaient des feuilles rouges barbouillées de sang. C'est le pays du chef qui accepte de manger les restes qui proviennent de la table de l'homme blanc. Le chef l'a fait de longues années, jusqu'au jour où les combattants sont venus le voir pour lui demander s'il était luimême un reste. (p. 118)

La collaboration avec la colonisation, motivée d'abord par l'appât du gain, l'envie de posséder des fusils comme les Blancs, puis par la nourriture - tandis que les terres 
laissées aux Noirs étaient de moins en moins fertiles - entraîne une rupture de plusieurs ordres : au sein de la collectivité, puisque les combattants et le chef servent des intérêts opposés (en ce sens, la collaboration rend l'intime étranger); au sein de la psyché, puisque le collaborateur - le chef, tout comme Chisaga - éprouve une honte et une culpabilité à dépendre du Blanc : il devient étranger à lui-même ; au sein de la nature, qui se gorge de sang (la terre, la montagne, les arbres, les feuilles: l'air, la terre): l'inquiétante étrangeté devient cosmique, et le désordre rompt l'équilibre avec l'environnement et la terre ${ }^{17}$.

Dès lors, c'est bien le colon qui devient objet de hantise, et la dénonciation a définitivement changé d'objet. Les familles des combattants-terroristes sont traquées et torturées. Marita, en premier lieu :

Marita, comment ils t'ont ramenée déchirée, en lambeaux! Comment ils t'ont ramenée, avec du sang à couler de tes oreilles! C'est difficile de tuer un être humain, Marita. Je l'ai vu de mes yeux. Si tu avais été une chèvre ou un chien, tu aurais déjà été morte quand ils t'ont jetée nue sur le sol boueux qui puait les vers et la bouse de vache. [...] Tu respirais, mais ton souffle s'échappait de la terre mousseuse qu'on t'avait enfoncée dans la bouche. Ils t'avaient brûlée avec divers objets, partout sur le corps, même aux endroits qu'on ne peut mentionner. (p. 99)

Est heimlich, d'après Freud, l'intime, le "cher ", les parties du corps voilées ${ }^{18}$. L'intime devient unheimlich lorsqu'il est dévoilé. L'expérience-limite de la torture, qui décidera Marita à partir lorsqu'elle aura retrouvé des forces, rend le corps étranger en ce qu'il est dévoilé, montré, exposé, exhibé, tandis qu'il est normalement caché. Il n'y a plus d'intime dans la violence extrême, et elle rend étrange le corps.

\section{Le seuil de la révolte}

Ce seuil de non-retour est expérimenté par deux personnages : Marita, torturée, et Janifa, violée.

Je préfère en rire, Marita, mais je pense que derrière ce que Chisaga m'a fait, il y avait ma mère. «Va nous chercher de l'eau! m'a-t-elle dit, le coin de l'œil scintillant comme une étoile arrachée au ciel par la main furieuse de dieu.» (p. 149)

«Et donc me voilà seule avec les blessures dont ma mère croit qu'elles me donneront du plaisir un jour. Du sang qui n'arrête pas de couler, et qui me fait mal à l'intérieur quand je revois le jour où ils t'ont ramenée, Marita, usée de tortures, usée comme un vieux chiffon, déchirée à l'intérieur, déchirée comme une guenille dont nul ne se soucie » (p. 151)

31 Ce sont les esprits qui finissent par raconter la fin de l'histoire, et annoncer que Marita est enfermée dans un asile. C'est le second chapitre où les "esprits", de la première Chimurenga, apparaissent, et ils se confondent clairement avec la figure d'un narrateur hétérodiégétique. Ce fait tout à fait singulier donne une profondeur à la voix des esprits très intéressante :

[The Spirits Speak] Elle s'agenouille pour dire que Marita lui en a beaucoup appris sur le corps des hommes et des femmes, des jeunes femmes qui un jour deviendront vieilles. Elle parle, elle parle, jusqu'à ce que des gens effrayants viennent la chercher dans la forêt, qu'elle puisse rester enfermée là où on garde ceux dont la tête est pleine d'insanités, des horreurs dont il faut protéger ceux qui ont la tête propre. Elle est avec les autres, qui se parlent tout seuls, sans attendre qu'on leur 
réponde. Ceux que des cordes solides attachent au lit, qu'ils n'aillent pas utiliser

leur trop plein de force pour se libérer. (p. 168) première est la proclamation d'une révolte future, venant donner sens à l'informe du corps meurtri : ce qu'annoncent les esprits-ancêtres (dans le premier chapitre «The spirits speak »). La seconde conséquence de cette profanation est l'indistinction du seuil de la folie : Janifa violée, avec la complicité de sa propre mère, est enfermée dans un asile, sans qu'il ne soit clair si une différence folie/raison existe bien : le motif du double touche petit à petit l'ensemble du collectif et du tissu social. Enfin, un troisième effet de cette profanation est la dénonciation du « silence » comme un fait de collaboration encore plus étrange que la folie de Janifa : «the silent tree », autrement dit, le fait de ne pas se révolter contre la situation coloniale, est lue comme paroxysme de l'inquiétant. Privé de parole, l'être devient amputé de sa capacité à faire sujet : il se dissout.

[The Spirits Speak - 1897 My Bones Fall] Je vais courir au sanctuaire de nos ancêtres et prononcer ces mots. [...] Je dirai : c'est la dernière fois que quelqu'un vous honorera comme il convient en ce sanctuaire, car les enfants de vos enfants ont succombé au silence. [...] Tous les lieux sacrés seront transformés en champs de labour et terrains de jeux, car vos enfants ont mangé à l'arbre du silence [the silent tree]. (p. 82)

... You can torture me, spread my bowels for the jackals to eat and tear them to pieces, mutilate my body with your anger throw my brains to the vultures, leave the remains of my body to the playground for your children to play with, cut my ears to decorate your own ears, cut my fingers use them to wipe your own sweat... my bones will rise in the spirit of war. They will sing war-songs with the fire of battle. (p. 50 Heinemann)

\section{Conclusion : la vallée de l'étrange}

Le roboticien Masahiro Mori a créé un graphique pour montrer à quel point «les créatures artificielles dont nous nous entourons doivent nous ressembler ou nous dissembler ${ }^{19}$. Ces conclusions pointent l'existence d'une "vallée de l'étrange ", à l'intérieur de laquelle les robots deviennent étrangement inquiétants s'ils ressemblent trop aux humains - ce qu'ils ne seront jamais. Est inquiétant ce qui est proche de soi, sans l'être tout à fait. Le robot ressemblant à un robot n'est pas inquiétant. Un robot trop anthropomorphe l'est terriblement.

Chenjerai Hove nous plonge dans cette "vallée de l'étrange " en montrant comment la guerre civile déchire l'ensemble des strates de la société, du village, de la famille, du couple, et des relations primaires mère-enfant, mère-fille. La mère vend sa fille pour quelques sous. Le propriétaire bat ses ouvriers. Le chef de village collabore. La nature se gorge de sang. Par la polyphonie narrative, le récit devient " exploratoire ${ }^{20}$ et la notion d'inquiétante étrangeté rend compte des différents seuils de malaise qu'expérimente le lecteur. La figure du terroriste incarne le premier seuil de ce malaise, de cette indécision : toujours raconté par les autres, mis en scène dans des scénarios multiples, il est celui de la maison qui revient semer l'horreur. Mais c'est très vite la place du collaborateur qui 
devient tout aussi inquiétante : il est le double, celui qui est moi sans être moi, qui me vend aux étrangers. Enfin, dernière figure, celle de la folie raisonnable : Janifa, Marita, rejetées dans les marges, et pourtant beaucoup plus familières que les figures de la mère ou de Chisaga, pourtant socialement acceptées.

C'est à un retournement du soupçon et du doute que nous invite Chenjerai Hove : affronter la "vallée de l'étrange », pour semer le doute d'une décomposition en marche, la destruction de l'empire ${ }^{21}$.

\section{BIBLIOGRAPHIE}

BEACH D., « An Innocent Woman, Unjustly Accused? Charwe, Medium of the Nehanda Mhondoro Spirit, and the 1896-97 Central Shona Rising in Zimbabwe », History in Africa (1998), p. 27-54.

BONHOMME J., Les voleurs de sexe. Anthropologie d'une rumeur africaine, Paris, 2009, Seuil.

DERRIDA J., Spectres de Marx : l'État de la dette, le travail du deuil et la nouvelle Internationale, Paris, 1993, Galilée.

FREUD S., L'Inquiétante étrangeté et autres essais, trad. Bertrand Féron, Nrf, Paris, 1985, Gallimard.

GARNIER X., L'éclat de la figure : étude sur l'antipersonnage de roman, Nouvelle Poétique

Comparatiste, Bruxelles, 2001, PIE-P. Lang.

GARNIER X., « Usages littéraires de la rumeur en Afrique », in La question des savoirs, Notre Librairie, 2001, p. 3-8.

GARNIER X., Sony Labou Tansi. Une écriture de la décomposition impériale, Paris, 2015, Karthala.

HOVE C., Bones, Harare, 1988, Baobab Books.

HOVE C., Shadows, Oxford, Portsmouth, Heinemann, 1992, Heinemann Educational Books, Inc.

HOVE C., Ossuaire, Arles, 1997, Actes Sud.

HOVE C., Ancêtres : roman, trad. Jean-Pierre Richard, Arles, 2002, Actes Sud.

KRISTEVA J., Étrangers à nous-mêmes, Paris, 1988, Fayard.

LAN D., Guns \& Rain: Guerrillas \& Spirit Mediums in Zimbabwe, Londres, Berkeley, J. Currey, 1985, University of California Press.

MORIN E., La rumeur d'Orléans, Paris, 1969, Seuil.

RANGER TO, Revolt in Southern Rhodesia, 1896-97: a study in African resistance, Evanston, 1967, Northwestern University Press.

RANGER TO, Writing Revolt: An Engagement with African Nationalism, 1957-67, Woodbridge, Harare, James Currey, 2013, Weaver Press.

SAMKANGE SJT, On Trial for my Country, Londres, 1966, Heinemann.

SAMKANGE SJT, Year of the Uprising, Londres, 1978, Heinemann. 
VEIT-WILD F., Teachers, Preachers, Non-believers: a Social History of Zimbabwean Literature, Londres, New York, 1992, Hans Zell Publishers.

\section{NOTES}

1. Sigmund Freud, L'Inquiétante étrangeté et autres essais, trad. Bertrand Féron, Nrf, Paris, 1985, Gallimard.

2. Parmi l'abondante historiographie sur cette guerre, signalons les deux ouvrages de David Lan, Guns \& Rain: Guerrillas \& Spirit Mediums in Zimbabwe, Londres, Berkeley, J. Currey, 1985, University of California Press ; T.O. Ranger, Writing Revolt: an Engagement with African Nationalism, 1957-67, Woodbridge, Harare, James Currey, 2013, Weaver Press.

3. Yvonne Vera, Nehanda, Harare, 1993, Baobab Books ; Yvonne Vera, The Stone Virgins, New York, 2003, Farrar, Straus and Giroux.

4. Stanlake John Thompson Samkange, On Trial for my Country, Londres, 1966, Heinemann.

5. Stanlake John Thompson Samkange, Year of the Uprising, Londres, 1978, Heinemann.

6. Hove (1956-2015) est traduit en français par Jean-Pierre Richard, et publié chez Actes Sud. Pour une analyse socio-historique des différentes générations d'écrivains au Zimbabwe, nous renvoyons à Flora Veit-Wild, Teachers, Preachers, Non-believers: a Social History of Zimbabwean Literature, Londres, New York, 1992, Hans Zell Publishers.

7. Chenjerai Hove, Ossuaire, Arles, 1997, Actes Sud ; et pour l'édition originale : Chenjerai Hove, Bones, Harare, 1988, Baobab Books.

8. Pour deux avis contradictoires sur le rôle des médiums pendant la première Chimurenga, voir TO Ranger, Revolt in Southern Rhodesia, 1896-97: a Study in African Resistance, Evanston, 1967, Northwestern University Press ; David N. Beach, «An Innocent Woman, Unjustly Accused? Charwe, Medium of the Nehanda Mhondoro Spirit, and the 1896-97 Central Shona Rising in Zimbabwe ", in History in Africa, 1998, p. 27-54.

9. Julia Kristeva, Étrangers à nous-mêmes, Paris, 1988, Fayard.

10. Yvonne Vera, Without a Name; and, Under the Tongue, New York, 2002, Farrar, Straus, and Giroux.

11. Xavier Garnier, «Usages littéraires de la rumeur en Afrique », in La question des savoirs, Notre Librairie, 2001, p. 3-8.

12. Julien Bonhomme, Les voleurs de sexe. Anthropologie d'une rumeur africaine, Paris, 2009, Seuil.

13. Edgar Morin, La rumeur d'Orléans, Paris, 1969, Seuil.

14. Xavier Garnier, L'éclat de la figure: étude sur l'antipersonnage de roman, Nouvelle Poétique Comparatiste, Bruxelles, 2001, PIE-P. Lang.

15. Jacques Derrida, Spectres de Marx : l'État de la dette, le travail du deuil et la nouvelle Internationale, Paris, 1993, Galilée.

16. Chenjerai Hove, Shadows, Oxford, Portsmouth, Heinemann, 1992, Heinemann Educational Books, Inc.

17. Voir Chenjerai Hove, Ancêtres : roman, trad. Jean-Pierre Richard, Arles, 2002, Actes Sud, p. 136 et suivantes, une prière en forme de chant adressée aux ancêtres, qui traite également de cet équilibre nature-bétail-société villageoise.

18. Freud, op.cit., p. 218.

19. Exposition « Persona - étrangement humain », Musée du Quai Branly, janvier-novembre 2016. 20. Pour reprendre l'expression de Chenjerai Hove, Jean-Pierre Richard, Ancêtres : roman, op. cit.

21. Comme chez Sony : Xavier Garnier, Sony Labou Tansi. Une écriture de la décomposition impériale, Paris, 2015, Karthala. 


\section{RÉSUMÉS}

Dans Ossuaire de Chenjerai Hove, la description de la guerre de libération nationale au Zimbabwe met en jeu de nombreux personnages d'ancêtres et des êtres fantômatiques. Nous analyserons l'inquiétante étrangeté que la guerre fait advenir dans ce roman ainsi que la décomposition de la trame narrative et des personnages face à la guerre civile.

In the novel Bones, written by Chenjerai Hove, ghostly figures and characters of the ancestors are linked to the description of the Liberation Struggle of Zimbabwe. I will analyze the depiction of the war and the way the unheimlich appears in the novel. I will also describe the decomposition of the narrative and of the characters facing the civil war.

\section{INDEX}

Mots-clés : Inquiétante étrangeté, spectres, Zimbabwe, guerre civile, Chenjerai Hove

Keywords : Unheimlich, ghosts, Zimbabwe, civil war, Chenjerai Hove

\section{AUTEUR}

\section{ELARA BERTHO}

Université Paris 3 - Sorbonne Nouvelle 